\title{
NOS3 is differentially expressed in hepatosplenic T-cell lymphoma.
}

Shahan Mamoor

shahanmamoor@gmail.com

Hepatosplenic T-cell lymphoma (HSTCL) is a rare peripheral T-cell lymphoma affecting the spleen and liver ${ }^{1}$. By mining published microarray datasets ${ }^{2-3}$ we found that the nitric oxide synthase 3 (NOS3) was among the genes most differentially expressed in HSTCL when compared to a different peripheral T-cell lymphoma, NKT Lymphoma. Analysis of a separate dataset revealed that when comparing HSTCL tissues and cell lines based on cytogenetic profile, NOS3 expression was associated with isochromosome 7q-positivity. NOS3 may be relevant to the biology of HSCTL.

Keywords: HSTCL, hepatosplenic T-cell lymphoma, peripheral T-cell lymphoma, NOS3, systems biology of HSTCL. 
Hepatosplenic T-cell lymphoma is a rare peripheral T-cell lymphoma usually featuring cells expressing the $\boldsymbol{\gamma} \boldsymbol{\delta} \mathrm{TCR}$, with median survival of close to eight months ${ }^{1}$.

Therapeutic options for patients with HSTCL are limited ${ }^{1}$. To understand HSTCL transcriptional behavior in an unbiased fashion, we mined published microarray datasets 2,3 and found that nitric oxide synthase 3 (NOS3) was differentially expressed in HSTCL when compared to a different peripheral T-cell lymphoma. NOS3 expression was also associated with cytogenetic status in patients with HSTCL, differentially expressed when comparing HSTCL cases based on the possession of isochromosome 7q. NOS3 may be pertinent to understanding molecular mechanisms that govern HSTCL.

\section{Methods}

We used datasets GSE $19067^{2}$ and GSE57942³ for this differential gene expression analysis of HSTCL. GSE19067 was generated using Affymetrix Human Genome U133 Plus 2.0 Array technology with $n=19$ NKT lymphoma samples and $n=4$ HSTCL samples, all pre-treated, fresh-frozen biopsy; analysis performed using platform GPL570. GSE57942 was generated using Agilent-014693 Human Genome CGH Microarray $244 \mathrm{~A}$ technology with $n=3$ Iso7q- HSTCL cases and $n=2$ Iso7q $+\mathrm{HSTCL}$ tissues and cell lines (1 Iso7q+ HSTCL case and 1 Iso7q+ HSTCL cell line, DERL-2); tissues were fresh-frozen lymphoma; analysis performed using platform GPL9128. The Benjamini and Hochberg method of $p$-value adjustment was used for ranking of differential expression; raw $p$-values were used for assessment of statistical significance 
of global differential expression. Log-transformation of data was auto-detected, and the NCBI generated category of platform annotation was used. A statistical test was performed to evaluate the significance of difference in NOS3 mRNA expression between NKT Iymphoma and HSTCL, as well as between Iso7q+ and Iso7q- HSTCL tissues and cell lines, using an unpaired two-tailed t-test with Welch's correction. All statistical analyses were performed using PRISM (8.4.0) Version 455.

\section{Results}

We mined published microarray datasets ${ }^{2,3}$ to understand HSTCL transcriptional behavior in an unbiased fashion by comparing HSTCL to a different type of peripheral Tcell lymphoma and comparing HSTCL cases based on cytogenetic profile.

NOS3 is differentially expressed in HSTCL when compared to NKT Lymphoma.

We found that nitric oxide synthase 3 (NOS3) was among the genes most differentially expressed when comparing tissues from 4 HSTCL patients to tissues from 19 patients with extranodal natural killer T-cell (NKT) lymphoma, also known as nasal NKT lymphoma ${ }^{2}$. When sorting each of the transcripts measured by microarray based on significance of change in expression between HSTCL and NKTL cases, NOS3 ranked 17 out of 54613 total transcripts. Differential expression of NOS3 in HSTCL as compared to NKTL was statistically significant (Table $1 ; p=4.89 \mathrm{E}-09$ ).

NOS3 is differentially expressed in Iso7q + HSTCL when compared to Iso7q- HSTCL.

Analysis of a separate dataset revealed that NOS3 was among the genes most differentially expressed when comparing tissues from 3 HSTCL patients negative for 
isochromosome 7q (Iso7q-) to 2 Iso7q+ HSTCL tissues: 1 patient positive for isochromosome $7 q(I s o 7 q+)$ and $1 \mathrm{Iso} q \mathrm{q}+\mathrm{HSTCL}$ cell line (DERL-2). When sorting each of the transcripts measured by microarray based on significance of change in expression between Iso7q- and Iso7q+ HSTCL tissues, NOS3 ranked 139 out of 235985 total transcripts. Differential expression of NOS3 in HSTCL based on Iso7q status in HSTCL was statistically significant (Table 2; $p=0.0067164$ ).

NOS3 is expressed at significantly higher levels in HSTCL when compared to NKT Lymphoma.

We obtained exact mRNA expression levels for NOS3 from each HSTCL and NKTL case 2 to directly compare NOS3 expression. We also performed a statistical test to evaluate whether the difference in NOS3 expression between HSTCL and NKTL tissues was statistically significant. NOS3 was expressed at higher levels in HSTCL as compared to NKTL, and this difference was statistically significant (Figure $1 ; p<0.0001$ ).

NOS3 is expressed at significantly higher levels in Iso7q+ HSTCL when compared to Iso7q- cases.

We also obtained exact mRNA expression levels for NOS3 from 3 Iso7q- HSTCL tissues and from 1 Iso7q + HSTCL tissue to directly compare NOS3 expression based on Iso7q status ${ }^{3}$. We also performed a statistical test to evaluate whether the difference in NOS3 expression between Iso7q- and Iso7q+ tissues and cell lines was statistically significant. NOS3 was expressed at higher levels in Iso7q+ HSTCL tissues and cell lines as compared to Iso7q- HSTCL tissues, and this difference was statistically significant (Figure 2; $p=0.0145$ ). 


\section{Discussion}

NOS3, or nitric oxide synthase $3^{4}$, functions in the generation of nitric oxide (NO), a signaling molecule whose function it is at the molecular level to stimulate the soluble form of guanylyl cyclase (sGC) and support the synthesis of a second messenger signaling molecule cyclic guanosine monophosphate (cGMP) ${ }^{5}$. cGMP can in turn activate cGMP-dependent protein kinases for signal transduction ${ }^{5}$. At the organismal level, among other functions, NO supports relaxation of smooth muscles, dilation state of blood vessels, or vascular tone, and motility of the intestines ${ }^{5}$. In the hematopoietic system, NOS3 is expressed in platelets and while NO is thought to be an inhibitor of platelet activation, in the context of low dose agonist platelet-activation, NOS3 promotes thrombogenesis 6 . Thus, in platelets, low levels of $\mathrm{NO}$ are thought to promote platelet aggregation while high levels of $\mathrm{NO}$ are thought to inhibit platelet aggregation 6 .

We could not identify literature describing a role for NOS3 in hepatosplenic T-cell lymphoma. In summary, an isoform of the enzyme that generates nitric oxide, nitric oxide synthase 3 (NOS3), is among the genes most differentially expressed in HSTCL when compared to a different peripheral T-cell lymphoma, NKT Lymphoma. Moreover, NOS3 is among the genes most differentially expressed in hepatosplenic T-cell lymphoma when comparing HSTCL tissues and cell line based on the presence or absence of the chromosomal aberration isochromosome $7 q$, a cytogenetic feature that defines many HSTCLs7 ${ }^{7}$. Higher expression of NOS3 in Iso7q+ HSTCL cases should be validated in larger patient datasets and the relevance, if any, of NOS3 expression in HSTCL survival, maintenance or progression can be assessed. 


\section{References}

1. Chanan-Khan, A., Islam, T., Alam, A., Miller, K.C., Gibbs, J., Barcos, M., Czuczman, M.S., Paplham, P., Hahn, T. and McCarthy, P., 2004. Long-term survival with allogeneic stem cell transplant and donor lymphocyte infusion following salvage therapy with anti-CD52 monoclonal antibody (Campath) in a patient with $\alpha / \beta$ hepatosplenic T-cell non-Hodgkin's lymphoma. Leukemia \& lymphoma, 45(8), pp.1673-1675.

2. Iqbal, J., Weisenburger, D.D., Chowdhury, A., Tsai, M.Y., Srivastava, G., Greiner, T.C., Kucuk, C., Deffenbacher, K., Vose, J., Smith, L. and Au, W.Y., 2011. Natural killer cell lymphoma shares strikingly similar molecular features with a group of non-hepatosplenic $\gamma \delta$ T-cell lymphoma and is highly sensitive to a novel aurora kinase A inhibitor in vitro. Leukemia, 25(2), pp.348-358.

3. Ferreiro, J.F., Rouhigharabaei, L., Urbankova, H., van der Krogt, J.A., Michaux, L., Shetty, S., Krenacs, L., Tousseyn, T., De Paepe, P., Uyttebroeck, A. and Verhoef, G., 2014. Integrative genomic and transcriptomic analysis identified candidate genes implicated in the pathogenesis of hepatosplenic Tcell lymphoma. PloS one, 9(7), p.e102977.

4. Robinson, L.J., Weremowicz, S., Morton, C.C. and Michel, T., 1994. Isolation and chromosomal localization of the human endothelial nitric oxide synthase (NOS3) gene. Genomics, 19(2), pp.350-357.

5. Schlossmann, J., Feil, R. and Hofmann, F., 2003. Signaling through $\mathrm{NO}$ and cGMP-dependent protein kinases. Annals of medicine, 35(1), pp.21-27.

6. Marjanovic, J.A., Li, Z., Stojanovic, A. and Du, X., 2005. Stimulatory roles of nitric-oxide synthase 3 and guanylyl cyclase in platelet activation. Journal of Biological Chemistry, 280(45), pp.37430-37438.

7. Alonsozana, E.L.C., Stamberg, J., Kumar, D., Jaffe, E.S., Medeiros, L.J., Frantz, C., Schiffer, C.A., O'Connell, B.A., Kerman, S., Stass, S.A. and Abruzzo, L.V., 1997. Isochromosome 7q: the primary cytogenetic abnormality in hepatosplenic yठ T cell lymphoma. Leukemia, 11(8), pp.1367-1372. 


\section{Rank ID p-value $t \quad$ B $\quad$ Gene Gene name \\ 17 \\ 205581_s_at \\ \begin{tabular}{l|l}
$4.89 E-09$ & 8.0498863
\end{tabular} \\ 10.40098 \\ NOS3 \\ nitric oxide synthase 3}

Table 1: NOS3 is differentially expressed in HSTCL when compared to NKT Lymphoma.

Rank of differential expression, probe ID, $p$-value of global differential expression, $t$, a moderated $t$-statistic, $B$, the log-odds of differential expression between HSTCL and NKTL, the gene and gene name are listed in this chart. 


\begin{tabular}{|r|l|l|l|l|l|l|}
\hline Rank & ID & p-value & t & B & Gene & Gene name \\
\hline 139 & A_14_P112011 & 0.0067164 & 3.64 & -4.44 & NOS3 & $\begin{array}{l}\text { nitric oxide synthase 3 } \\
\text { (endothelial cell) }\end{array}$ \\
\hline
\end{tabular}

Table 2: NOS3 is differentially expressed in Iso7q+ HSTCL when compared to Iso7q- HSTCL.

Rank of differential expression, probe ID, p-value of global differential expression, $\mathrm{t}$, a moderated t-statistic, $\mathrm{B}$, the log-odds of differential expression between HSTCL and NKTL, the gene and gene name are listed in this chart. 


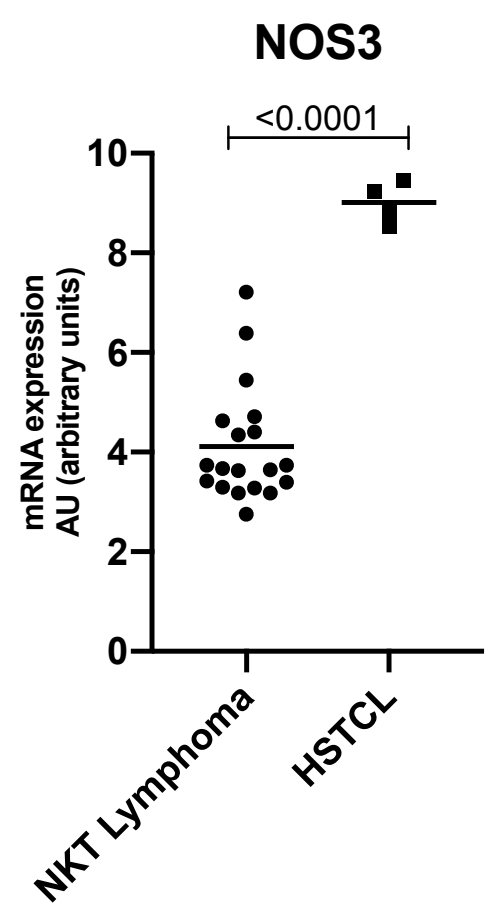

Fiqure 1: NOS3 is expressed at significantly higher levels in HSTCL when compared to NKT Lymphoma.

The mRNA expression level of NOS3 in NKT lymphomas (left; $n=19$ ) and HSTCL (right; $n=4$ ) is graphically represented with mean mRNA values marked and the result of a statistical test evaluating significance of difference in NOS3 expression between the groups, a $p$-value, listed above. 


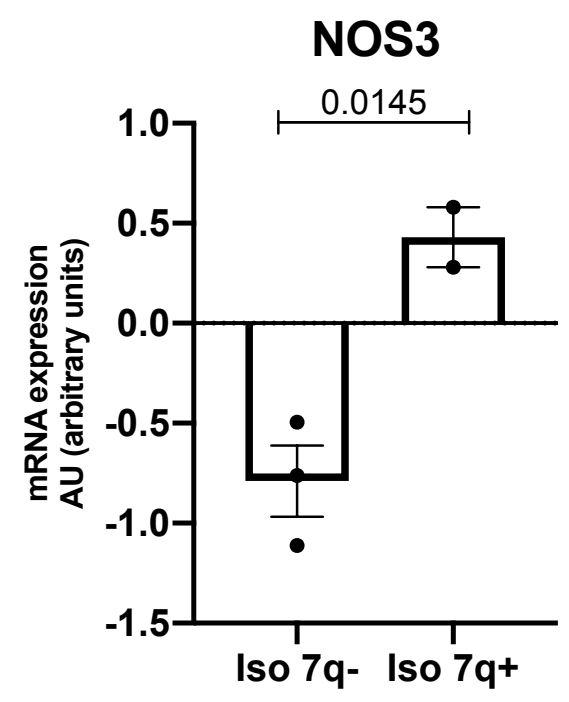

Figure 2: NOS3 is expressed at significantly higher levels in Iso7q+ HSTCL when compared to Iso7q- cases.

The mRNA expression level of NOS3 in Iso7q- HSTCL (left; $n=3$ cases) and in Iso7q+ HSTCL (right; $n=2 ; n=1$ case and $n=1$ HSTCL cell line) is graphically represented with mean mRNA values marked and the result of a statistical test evaluating significance of difference in NOS3 expression between the groups, a $p$-value, listed above. 